\title{
Effects of Kettlebell Swing vs. Explosive Deadlift Training on Strength and Power
}

\author{
Matthew R. Maulit \\ Center for Sport Performance and Human Performance Lab, Department of Kinesiology, California State University, Fullerton, CA, USA \\ 800 N. State College Blvd., Fullerton, CA 92834-6870, USA \\ E-mail: mrmaulit@gmail.com \\ David C. Archer
}

Center for Sport Performance and Human Performance Lab, Department of Kinesiology, California State University, Fullerton, CA, USA 800 N. State College Blvd., Fullerton, CA 92834-6870, USA

E-mail: dvdarcher@csu.fullerton.edu

Whitney D. Leyva

Center for Sport Performance and Human Performance Lab, Department of Kinesiology, California State University, Fullerton, CA, USA 800 N. State College Blvd., Fullerton, CA 92834-6870, USA

Email: whitleyva@gmail.com

Cameron N. Munger

Center for Sport Performance and Human Performance Lab, Department of Kinesiology, California State University, Fullerton, CA, USA 800 N. State College Blvd., Fullerton, CA 92834-6870, USA

Email: cammunger@gmail.com

Megan A. Wong

Center for Sport Performance and Human Performance Lab, Department of Kinesiology, California State University, Fullerton, CA, USA 800 N. State College Blvd., Fullerton, CA 92834-6870, USA

Email: mwong52493@csu.fullerton.edu

Lee E. Brown (Corresponding author)

Center for Sport Performance and Human Performance Lab, Department of Kinesiology, California State University, Fullerton, CA, USA 800 N. State College Blvd., Fullerton, CA 92834-6870, USA

E-mail: leebrown@fullerton.edu

Jared W. Coburn

Center for Sport Performance and Human Performance Lab, Department of Kinesiology, California State University, Fullerton, CA, USA 800 N. State College Blvd., Fullerton, CA 92834-6870, USA

E-mail: jcoburn@fullerton.edu

Andrew J. Galpin

Center for Sport Performance and Human Performance Lab, Department of Kinesiology, California State University, Fullerton, CA, USA 800 N. State College Blvd., Fullerton, CA 92834-6870, USA

E-mail: agalpin@fullerton.edu

Received: 9-12- 2016

doi:10.7575/aiac.ijkss.v.5n.1p.1
Accepted: 25-01- 2017

Published: 31-01- 2017

URL: http://dx.doi.org/10.7575/aiac.ijkss.v.5n.1p.1

\section{Abstract}

Background: Recent research has compared explosive deadlift to kettlebell training observing their effects on strength. The kettlebell swing is a popular practical exercise as it shares share a hip hinge movement with the explosive deadlift, but the two have not been compared. Objectives: The purpose of this study was to compare the 
effects of kettlebell swing vs. explosive deadlift training on strength and power. Methods: Thirty-one recreationally resistance-trained men $($ age $=23.1 \pm 2.3$ years, height $=175.5 \pm 6.6 \mathrm{~cm}$, mass $=83.9 \pm 13.8 \mathrm{~kg}, 1 \mathrm{RM}$ deadlift $=$ $159.9 \pm 31.7 \mathrm{~kg}$ ) were randomly assigned to one of two groups [kettlebell swing group (KBG) $n=15$, or explosive deadlift group (EDLG) $\mathrm{n}=16$ ]. Vertical jump height, isometric mid-thigh pull (MTP), and 1RM deadlift were measured pre and post training. Both groups trained twice per week for 4 weeks. Volume and load were increased after the first 2 weeks of training. Results: A 2 (time) x 2 (group) mixed factor ANOVA revealed a significant $(\mathrm{P}<0.05)$ increase in deadlift $1 \mathrm{RM}$ (pre: $159.9 \pm 31.7 \mathrm{~kg}$, post: $168.9 \pm 31.8 \mathrm{~kg}$ ) and vertical jump height (pre: $56.6 \pm$ $9.9 \mathrm{~cm}$, post: $57.9 \pm 9.7 \mathrm{~cm}$ ) for both groups, but were not significantly different between groups. There were no significant changes in MTP. Conclusions: Strength and conditioning professionals may use both kettlebell swings and explosive deadlifts to increase deadlift strength and vertical jump power.

Keywords: vertical jump, isometric mid-thigh pull, hip hinge.

\section{Introduction}

Power production, which is the product of force and velocity, is arguably the most important characteristic required of athletes in numerous sports. The ability to produce force quickly is essential in explosive movements like jumping, throwing, and sprinting. Hinge exercises, those involving flexion or extension at the hip and knee, significantly affect the power produced in the aforementioned movements. Many methods for improving rate of force production in the hip hinge pattern exist, but the rise in kettlebell training popularity poses both specific and relevant questions. For this reason, traditional hip hinge exercises like the barbell deadlift, while previously shown to increase peak force and power, are now being compared to exercises like the kettlebell swing (Blatnik et al., 2014; Otto, Coburn, Brown, \& Spiering, 2012; Swinton, Stewart, Keogh, Agouris, \& Lloyd, 2011).

It has previously been shown that kettlebell swing training improves rapid lower body force production, but not peak force as much as traditional weightlifting movements (Lake \& Lauder, 2012; Otto et al., 2012). Results such as these are no surprise considering the kettlebell lacks the capacity for high loads in comparison to a loaded barbell. However, speed of movement during the kettlebell swing may be more specific to athletic movements making it an ideal exercise for explosive strength development. Based on the concepts of velocity specific training, the kettlebell swing may have an advantage over the explosive deadlift in enhancing performance. However, the ability to optimally and accurately load a barbell for the purposes of power training adaptations has not been achieved with the kettlebell swing. In response to this, the current study sought to examine loaded kettlebell training for the purposes of power production. The isometric mid-thigh pull highly correlates with weightlifting movements emulating a similar hip extension pattern seen during performance of the kettlebell swing therefore force output during this test was used for load determination (Beckham et al., 2013). Additionally, progressive overload in previous kettlebell research was achieved through either uniform alterations to load across subjects, by increasing volume of sets and repetitions, or increasing work to rest ratios (Jay et al., 2011; Jay et al., 2013; Lake \& Lauder, 2012; Manocchia, Spierer, Lufkin, Minichiello, \& Castro, 2013; Otto et al., 2012). The present study sought to achieve overload through increases in intensity and decreases in volume. How the improvements in power output from kettlebell training compare with more traditional exercises like the deadlift is yet unclear. Therefore, the purpose of this study was to compare the effects of four weeks of kettlebell swing versus explosive deadlift training on performance.

\section{Methods}

\subsection{Study Design}

Participants were randomly assigned to four weeks of either kettlebell swing or explosive deadlift training. Testing was performed prior to and after the 4-week training protocol. Performance variables were measured via the countermovement vertical jump, maximal isometric mid-thigh pull, and 1RM deadlift. Progressive overload was achieved through increases in load.

\subsection{Participants}

Thirty-one males (age $=23.13 \pm 2.38$ years, height $=175.52 \pm 6.66 \mathrm{~cm}$, weight $=83.97 \pm 13.85 \mathrm{~kg}$ ) with at least one year resistance training experience, and experience with both exercises (two times per week) volunteered. Experimental procedures were approved by the University Institutional Review Board for Human Subjects, and all participants read and signed an informed consent prior to participation. The first week entailed two laboratory visits, which included testing and familiarization with the two exercises. Familiarization specifically included three sets of five repetitions of each exercise style. Upon completion of the first week, subjects were randomly assigned to either the explosive deadlift, or kettlebell swing training groups. Participants were instructed not to perform either of the exercises outside of the research study. Each group trained twice per week for four weeks with 48-72 hours between visits. Following the 4-week training protocol, subjects were retested on the same performance measures as week 1. Dietary instructions were to adhere to their normal eating habits. Subjects were excluded if they were presently using any dietary or performance enhancing substances.

\subsection{Procedures}

Prior to testing, each subject's height and mass were measured using a stadiometer (752KL, Seca; Ontario, CA, USA) and a digital scale (ES200L, Ohaus Corporation; Pinebrook, NJ, USA). They also performed a dynamic warm-up before testing and each training session, which included a lunge with torso rotation, knee hugs, walking 
hamstring stretch, and Frankenstein's (forceful kicks with a toe touch at the top of the kick) for a distance of 10 meters each.

\subsection{Vertical Jump}

Vertical jump height was measured using a jump station (EPIC, JS; Lincoln, NE, USA). They were performed on an AMTI force plate (Advanced Mechanical, Inc.; Watertown, MA, USA) for the measurement of peak power, and ground reaction force. Each participant performed three countermovement vertical jumps with 1-minute rest intervals. The highest score of the three jumps was recorded and used for analysis.

\subsection{One Repetition Maximum Testing}

Pre- and posttest 1RM deadlift testing was performed by having participants stand with their feet shoulder-width apart, and toes pointed moderately outward. Squatting down, they grasped the bar with a pronated or alternated grip, and knees inside fully extended arms. The use of lifting straps (ROGUE Fitness, Ohio Lifting Straps; Columbus, $\mathrm{OH}$, USA) was required regardless of grip style. Before movement began the bar rested approximately 1 inch from the shins over the distal end of the metatarsals. Upper body position included a flat or slightly arched back, upright chest, neck slightly hyperextended, and eyes looking forward. Movement began by extending the hips and knees while maintaining a flat back. Bar path remained as close to the shins as possible until past the knees at which point the hips came forward and the bar came into contact with the thighs. Once they reached full knee extension and were standing completely upright the downward movement phase began. Downward movement was defined by hip and knee flexion, and maintaining a flat back position until the weight reached the floor. Exercise technique was crossreferenced with guidelines of the National Strength and Conditioning Association (NSCA). The warm-up protocol included 10 reps at 50\% estimated 1RM, 5 reps at 70\%1RM, 3 reps at 80\%1RM, and 1 rep at 90\%1RM (Archer et al., 2016). Following the warm-up, participants were allotted three attempts (3-5 minutes rest between attempts) to achieve their 1RM.

2.6 Maximal Isometric Mid-Thigh Pull

Three maximal isometric mid-thigh pulls were performed on the same force plate pre- and posttest. The participant stepped into a power rack (ROGUE Fitness; Columbus, OH, USA) customized for the placement of a barbell at any height. Lifting straps were utilized throughout testing as the participants grasped the bar with either a pronated or alternated grip. Knee angle was assessed with a handheld goniometer to ensure angles were between $125-135^{\circ}$, and body position was similar to the second pull of the snatch. Each participant first performed three warm up pulls at $50 \%, 75 \%$, and $100 \%$ of maximal effort, but was instructed to be as still as possible in order to minimize force output before the initiation of the test. Upon completion of the warm up pulls, three maximal pulls were performed with one minute rest intervals. These methods previously elicited RFD and peak force scores, which significantly correlated to weightlifting performance involving hip extension similar to the exercises included in the present study (Beckham et al., 2013).

\subsection{Kettlebell Swing Training}

Participants in the kettlebell swing group (KBG) trained with a KettleClamp (KettleClamp; Simpsonville, SC, USA) handle attached to a dumbbell with combined weight approximately 10-12.5\% of peak delta force produced during the maximal isometric mid-thigh pull. The kettlebell swing began by grasping the kettlebell with a two-handed pronated grip, elbows locked out, and feet slightly outside shoulder width. The back was flat, and the neck was neutral or slightly extended throughout the movement. Feet were planted in the ground from toe to heal. During the downward movement the kettlebell passed above the knee as the knees and hips flexed. Knee flexion at the bottom of the swing ranged between $140-145^{\circ}$. The upward swing began as the hips and knees extended rapidly until the body formed a straight line at the top of the swing. Arms were fully extended anteriorly, and the kettlebell did not pass chin height. A linear periodization training model was used and began with 4 x 5 (sets x reps) in weeks 2-3 with a load of $10 \%$ peak delta force from the isometric mid-thigh pull, and concluded in weeks $4-5$ using a $6 \times 4$ setup at $12.5 \%$. Researcher instructions reiterated the importance that all swings be performed at maximal velocity, but never at the sacrifice of proper technique. By increasing intensity and altering volume, progressive overload was achieved.

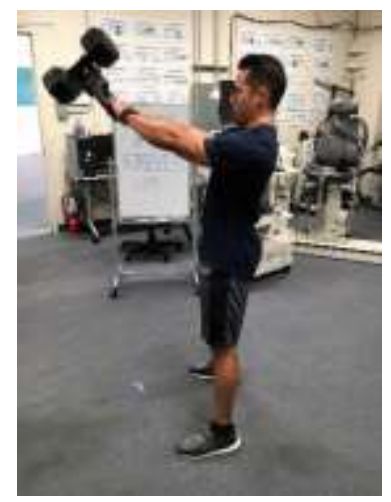

Figure 1. Kettlebell Swing 


\subsection{Explosive Deadlift Training}

The explosive deadlift training group (EDLG) lifted a barbell loaded with $30 \%-40 \%$ of their 1 RM as previous research has shown peak power readings at this intensity with this specific exercise (Kaneko, Fuchimoto, Toji, \& Suei, 1983; Swinton et al., 2011). Technique of the explosive deadlift is similar to the standard deadlift (detailed in the "One Repetition Maximum" section under "Procedures"), but the participant was instructed to extend the hips and knees at maximal velocities during the upward phase. Feet remained planted throughout the entirety of the exercise. Movement was slower during the downward movement to ensure subsequent explosive lifts began with proper form and velocity. Linear periodization was also implemented in this training as it began in weeks 1-3 utilizing a 4 x 5 protocol at 30\%1RM, and concluded in weeks 4-5 with a 6 x 4 setup at 40\%1RM. Similar to the kettlebell training group, progressive overload was obtained through an increase in intensity and an alteration in volume.

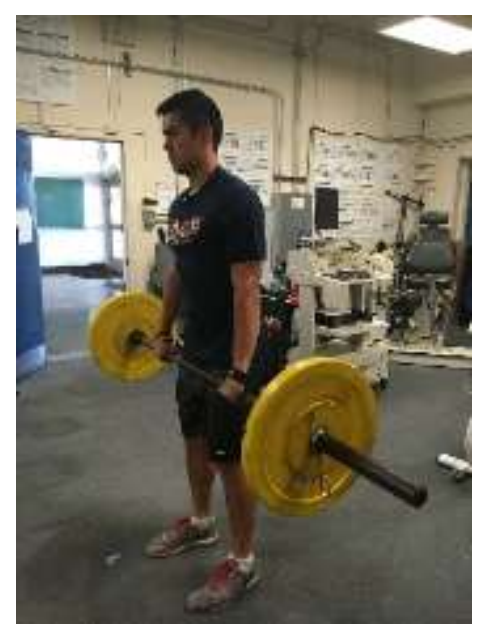

Figure 2. Explosive Deadlift

\subsection{Statistical Analyses}

Multiple 2 (time) x 2 (group) mixed factor ANOVAs were utilized to compare vertical jump height, peak power (PP), and ground reaction force (GRF), 1RM deadlift, maximal isometric mid-thigh pull rolling rate of force development (RRFD), and delta force. Upon a significant interaction, a follow-up simple ANOVA was used. An alpha level of 0.05 was used to determine significance.

\section{Results}

\subsection{RM Deadlift Scores}

For deadlift 1RM, there was no significant interaction or main effect for group. There was a significant main effect for time, where post was greater than pre (see Table 1).

Table 1. 1RM Deadlift. Mean (SD) of one repetition maximum (1RM) deadlift (kg) between kettlebell swing (KBG) and explosive deadlift groups (EDLG) and as a total group (grand mean)

\begin{tabular}{lcccccc}
\hline & \multicolumn{2}{c}{ KBG } & \multicolumn{2}{c}{ EDLG } & \multicolumn{2}{c}{ Grand Mean } \\
\cline { 2 - 7 } Variable & Pre & Post & Pre & Post & Pre & Post \\
\hline \multirow{2}{*}{ Deadlift 1RM } & $164.8(32.4)$ & $173.0(32.4)$ & $155.2(31.4)$ & $165.2(31.8)$ & $159.9(31.7)$ & $168.9(31.8)^{*}$ \\
\hline
\end{tabular}

* Significantly greater than pre

\subsection{Vertical Jump Scores}

- For vertical jump height $(\mathrm{cm})$, there was no significant interaction or main effect for group. There was a significant main effect for time, where post was greater than pre (see Table 2).

- For vertical jump peak power (W), there was no significant interaction or main effect for time. There was a significant main effect for group, where KBG was greater than EDLG (see Table 2).

- For vertical jump GRF, there was no significant interaction or main effect for time. There was a significant main effect for group, where KBG was greater than EDLG. (see Table 2). 
Table 2. Vertical Jump Variables. Mean (SD) of Vertical Jump (cm), Ground Reaction Force (N), Peak Power (W), Between Kettlebell Swing Group (KBG), and Explosive Deadlift Group (EDLG) and as a total group (grand mean)

\begin{tabular}{lllllllll}
\hline & \multicolumn{1}{l}{ KBG } & \multicolumn{3}{c}{ EDLG } & \multicolumn{3}{c}{ Grand Mean } \\
\cline { 2 - 9 } & Pre & Post & Combined & Pre & Post & Combined & Pre & Post \\
\hline \multirow{2}{*}{ VJ } & 55.8 & 56.9 & 56.3 & 57.3 & 58.8 & 58.0 & 56.6 & $57.9^{*}$ \\
& $(9.9)$ & $(9.7)$ & $(9.8)$ & $(10.1)$ & $(10.0)$ & $(10.0)$ & $(9.9)$ & $(9.7)$ \\
& & & & & & & & \\
GRF & 2416.4 & 2378.8 & $2397.6^{* *}$ & 2045.5 & 2002.6 & 2024.1 & 2230.9 & 2190,7 \\
& $(539.5)$ & $(464.3)$ & $(501.9)$ & $(299.9)$ & $(274.3)$ & $(287.1)$ & $(419.7)$ & $(369.3)$ \\
PP & 5672.7 & 5717.4 & $5695.1^{* *}$ & 4908.4 & 4781.1 & 4844.7 & 5290.5 & 5249.2 \\
& $(1163.1)$ & $(1160.7)$ & $(1161.9)$ & $(959.1)$ & $(882.8)$ & $(920.9)$ & $(1061.1)$ & $(1021.7)$ \\
\hline
\end{tabular}

* Significantly greater than pre

** Significantly greater than EDLG

\subsection{Maximal Isometric MTP Scores}

- For isometric MTP delta force, there was no significant interaction or main effects for group or time (see Table 3).

- For isometric MTP rolling rate of force development (RRFD), there was a significant interaction of group and time (see Table 3). KBG had significantly greater RRFD at $50 \mathrm{~ms}$ than $250 \mathrm{~ms}, 100 \mathrm{~ms}$ than $250 \mathrm{~ms}$, $150 \mathrm{~ms}$ than 200 and $250 \mathrm{~ms}$, and $200 \mathrm{~ms}$ than $250 \mathrm{~ms}$ (see Table 3). EDLG had significantly greater RRFD at $150 \mathrm{~ms}$ than $250 \mathrm{~ms}$, and $200 \mathrm{~ms}$ than $250 \mathrm{~ms}$ (see Table 3).

Table 3. Isometric Mid-Thigh Pull Variables. Mean (SD) of Isometric Mid-Thigh Pull (MTP) Delta Force (N), Rolling Rate of Force Development (RRFD N/ms) Between Kettlebell Swing Group (KBG) and Explosive Deadlift Group (EDLG) and as a total group (combined)

\begin{tabular}{|c|c|c|c|c|c|c|}
\hline \multirow[b]{2}{*}{ Variable } & \multicolumn{3}{|c|}{ KBG } & \multicolumn{3}{|c|}{ EDLG } \\
\hline & Pre & Post & Combined & Pre & Post & Combined \\
\hline Delta Force & $\begin{array}{l}2576.7 \\
(447.3)\end{array}$ & $\begin{array}{l}2480.1 \\
(595.2)\end{array}$ & $\begin{array}{l}2528.4 \\
(521.2)\end{array}$ & $\begin{array}{l}2401.8 \\
(579.7)\end{array}$ & $\begin{array}{l}2288.7 \\
(630.8)\end{array}$ & $\begin{array}{c}2345.2 \\
(605.2)\end{array}$ \\
\hline RRFDpeak50ms & $\begin{array}{l}12015.6 \\
(5243.5)\end{array}$ & $\begin{array}{l}11702.8 \\
(4170.8)\end{array}$ & $\begin{array}{l}11859.2^{*} \\
(4204.4)\end{array}$ & $\begin{array}{c}8906.5 \\
(6113.3)\end{array}$ & $\begin{array}{c}7529.1 \\
(4742.9)\end{array}$ & $\begin{array}{c}8217.8 \\
(5242.7)\end{array}$ \\
\hline $\begin{array}{l}\text { RRFDpeak100 } \\
\mathrm{ms}\end{array}$ & $\begin{array}{l}10921.8 \\
(4325.8)\end{array}$ & $\begin{array}{l}11062.6 \\
(3383.8)\end{array}$ & $\begin{array}{l}10992.2^{*} \\
(3531.0)\end{array}$ & $\begin{array}{c}9433.5 \\
(3768.6)\end{array}$ & $\begin{array}{c}8518.6 \\
(3902.0)\end{array}$ & $\begin{array}{l}8976.0 \\
(3563.9)\end{array}$ \\
\hline $\begin{array}{l}\text { RRFDpeak150 } \\
\mathrm{ms}\end{array}$ & $\begin{array}{l}10673.0 \\
(2871.6)\end{array}$ & $\begin{array}{l}11793.6 \\
(3413.2)\end{array}$ & $\begin{array}{c}11233.3 * * \\
(2703.3)\end{array}$ & $\begin{array}{l}10260.4 \\
(3704.0)\end{array}$ & $\begin{array}{c}9139.0 \\
(3796.4)\end{array}$ & $\begin{array}{l}9699.7^{*} \\
(3466.5)\end{array}$ \\
\hline $\begin{array}{l}\text { RRFDpeak200 } \\
\mathrm{ms}\end{array}$ & $\begin{array}{c}9731.5 \\
(1929.2)\end{array}$ & $\begin{array}{l}10187.1 \\
(2563.4)\end{array}$ & $\begin{array}{l}9959.3^{*} \\
(2045.1)\end{array}$ & $\begin{array}{c}9254.7 \\
(2569.5)\end{array}$ & $\begin{array}{c}8619.0 \\
(2819.4)\end{array}$ & $\begin{array}{l}8936.9^{*} \\
(2463.5)\end{array}$ \\
\hline $\begin{array}{l}\text { RRFDpeak } 250 \\
\text { ms }\end{array}$ & $\begin{array}{c}8313.0 \\
(1637.8)\end{array}$ & $\begin{array}{c}8407.9 \\
(1961.6)\end{array}$ & $\begin{array}{c}8360.5 \\
(1626.6)\end{array}$ & $\begin{array}{c}7869.8 \\
(1900.4)\end{array}$ & $\begin{array}{c}7337.5 \\
(2186.3)\end{array}$ & $\begin{array}{c}7603.7 \\
(1921.4)\end{array}$ \\
\hline
\end{tabular}

* Significantly greater than RRFDpeak250ms

** Significantly greater than RRFDpeak200ms and RRFDpeak250ms

\section{Discussion}

The purpose of this study was to compare the effects of kettlebell swing versus explosive deadlift training on performance. The main findings were increases in 1RM deadlift, and vertical jump height for both training groups which may be due to a combination of neural and biomechanical adaptations following periodized velocity specific resistance training (Hill, 1938; Kaneko et al., 1983; Tillin, Pain, \& Folland, 2012; Wilson, Newton, Murphy, \& Humphries, 1993). The current study affirms velocity specific resistance training of the hip hinge movement can lead to increases in performance such as the vertical jump (Adams, O'Shea, O'Shea, \& Climstein, 1992). McBride 
and Triplett previously found that velocity of training and load lifted are highly correlated with the development of strength and power (McBride, Triplett-McBride, Davie, \& Newton, 2002). Essentially, faster movements increase velocity and slower movements increase strength. This concept was applied to the current study when identifying a training load for the kettlebell swing as no previous research had attempted to identify proper training loads for power development with this implement. A kettlebell weight of 10-12.5\% of the maximal isometric MTP was used to allow for fast, forceful movement of the implement. Previous studies utilizing 30-40\%1RM in the deadlift elicited increases in velocity, and this was also confirmed in the current study (Swinton et al., 2011). Additionally, previous research on periodized kettlebell training did not manipulate load as in the current study. Previous studies have utilized various approaches to manage intensity including an RPE scale, work to rest ratios, difficulty of exercise choice, participant's mass, or simply uniformly increased load for all participants (Jay et al., 2011; Jay et al., 2013; Manocchia et al., 2013). Otto et al. utilized a linear periodization program over the course of a 6-week training study comparing traditional weightlifting and kettlebells, in which progressive overload was achieved through increases in volume and velocity of movement (Otto et al., 2012). Similar to the present study, they found that both training groups significantly increased vertical jump height. The current study also followed a traditional linear periodization protocol, but progressive overload was achieved through increases in load with decreases in volume. The starting kettlebell load was $10 \%$ of a maximal isometric MTP for the first two weeks of training, and was increased to $12.5 \%$ for the final two weeks. Utilization of a commercial clamp allowed for customization of the kettlebell load for each participant. Since increases in vertical jump were realized the present study confirms that manipulation of kettlebell swing load, and a reduction in volume over the course of a periodized training program can lead to performance increases. Therefore the results of the current study provide an alternate method to the increases in volume and velocity of movement used by Otto et al. previously (Otto et al., 2012). What was unexpected in the current study was the ability of the KBG to increase their 1RM deadlift. There are a number of reasons why this could have occurred with the most likely explanation being greater neural activation. Adams found that training with 70$100 \% 1 \mathrm{RM}$ loads increased power of the vertical jump (Adams et al., 1992). In the current study, essentially the opposite was found: power training increased strength performance. Aagaard examined heavy resistance training and its effects on RFD and impulse (Aagaard, Simonsen, Andersen, Magnusson, \& Dyhre-Poulsen, 2002). Both significantly increased and correlated highly with EMG increases in the initial phase of muscle contraction between 0-200 ms. Aagaard concluded that while velocity was low with high resistance, attempting to lift the weight as fast as possible led to increased RFD. Therefore, it may be that while loads were low in the current study, participants' attempt to move as fast as possible may have led to increased motor unit activation. Similarly, previous studies comparing kettlebell training to traditional weightlifting have shown transfer to other exercises. Kettlebell exercise has been found to increase 1RM back squat, 1RM power clean, 3RM bench press, and 3RM clean and jerk (Aagaard et al., 2002; Manocchia et al., 2013). A biomechanical analysis performed by McGill demonstrated similar hip hinge movement patterns between various kettlebell exercises, and traditional weightlifting and powerlifting movements (McGill \& Marshall, 2012). Therefore, these shared movement patterns could contribute to the increase in 1RM deadlift over time. Variation within a periodized resistance training program is also essential to positive adaptations and increases in performance (Garhammer, 1979). According to Selye's general adaptive syndrome (GAS) a new stressor or stimulus must occur to lead to adaptation (Selye, 1956). Without the introduction of new stimuli into a training program an athlete can experience staleness syndrome, in which their body grows accustomed to the stresses consistently placed upon it. Since the participants recruited for this study had previous resistance training experience, it is possible there was a novel training effect caused by the introduction of new exercises into their resistance training program. New stimuli send the body into an alarm phase thereby causing the body to resist these new stresses thus leading to adaptation (Selye, 1956). Therefore, novel training could have also assisted in the participants' increases in performance in the 1RM DL and vertical jump.

\section{Conclusion}

Both the KBG and EDLG improved explosively in the vertical jump and forcefully in the deadlift. Therefore, due to the short training duration, it can be reasonably assumed that neural, hypertrophic, or biomechanical factors were primarily responsible for the improvements. Results of the current study demonstrate and reinforce that power and strength training affect each other positively (Aagaard et al., 2002; Adams et al., 1992; Hill, 1938). Future research should attempt to identify proper loads for the kettlebell, and compare different hip hinge exercises to the kettlebell swing. Strength and conditioning professionals can utilize both the kettlebell swing and explosive deadlift to increase power and strength.

\section{References}

Aagaard, P., Simonsen, E. B., Andersen, J. L., Magnusson, P., \& Dyhre-Poulsen, P. (2002). Increased rate of force development and neural drive of human skeletal muscle following resistance training. Journal of applied physiology, 93(4), 1318-1326.

Adams, K., O'Shea, J. P., O'Shea, K. L., \& Climstein, M. (1992). The Effect of Six Weeks of Squat, Plyometric and Squat-Plyometric Training on Power Production. The Journal of Strength \& Conditioning Research, 6(1), 36-41. 
Archer, D. C., Brown, L. E., Coburn, J. W., Galpin, A. J., Drouet, P. C., Leyva, W. D., et al. (2016). Effects of Short-Term Jump Squat Training With and Without Chains on Strength and Power in Recreational Lifters. International Journal of Kinesiology and Sports Science, 4(4), 18-24.

Beckham, G., Mizuguchi, S., Carter, C., Sato, K., Ramsey, M., Lamont, H., et al. (2013). Relationships of isometric mid-thigh pull variables to weightlifting performance. Journal of Sports Med and Physical Fitness, 53(5), 573-581.

Blatnik, J. A., Goodman, C. L., Capps, C. R., Awelewa, O. O., Triplett, T. N., Erickson, T. M., et al. (2014). Effect of Load on Peak Power of the Bar, Body and System during the Deadlift. Journal of Sports Science and Medicine, 13(3), 511-515.

Garhammer, J. (1979). Periodization of strength training for athletes. Track Tech, 73, 2398-2399.

Hill, A. (1938). The heat of shortening and the dynamic constants of muscle. Proceedings of the Royal Society of London B: Biological Sciences, 126(843), 136-195.

Jay, K., Frisch, D., Hansen, K., Zebis, M. K., Andersen, C. H., Mortensen, O. S., et al. (2011). Kettlebell training for musculoskeletal and cardiovascular health: a randomized controlled trial. Scandinavian journal of work, environment \& health, 196-203.

Jay, K., Jakobsen, M. D., Sundstrup, E., Skotte, J. H., Jørgensen, M. B., Andersen, C. H., et al. (2013). Effects of kettlebell training on postural coordination and jump performance: a randomized controlled trial. The Journal of Strength \& Conditioning Research, 27(5), 1202-1209.

Kaneko, M., Fuchimoto, T., Toji, H., \& Suei, K. (1983). Training effect of different loads on the force-velocity relationship and mechanical power output in human muscle. Scandinavian Journal of Sports Science, 5(2), 50-55.

Lake, J. P., \& Lauder, M. A. (2012). Kettlebell swing training improves maximal and explosive strength. The Journal of Strength \& Conditioning Research, 26(8), 2228-2233.

Manocchia, P., Spierer, D. K., Lufkin, A. K., Minichiello, J., \& Castro, J. (2013). Transference of kettlebell training to strength, power, and endurance. The Journal of Strength \& Conditioning Research, 27(2), 477-484.

McBride, J. M., Triplett-McBride, T., Davie, A., \& Newton, R. U. (2002). The effect of heavy-vs. light-load jump squats on the development of strength, power, and speed. The Journal of Strength \& Conditioning Research, 16(1), 75-82.

McGill, S. M., \& Marshall, L. W. (2012). Kettlebell swing, snatch, and bottoms-up carry: back and hip muscle activation, motion, and low back loads. The Journal of Strength \& Conditioning Research, 26(1), 16-27.

Otto, W. H., Coburn, J. W., Brown, L. E., \& Spiering, B. A. (2012). Effects of weightlifting vs. kettlebell training on vertical jump, strength, and body composition. The Journal of Strength \& Conditioning Research, 26(5), 1199-1202.

Selye, H. (1956). The Stress of Life. New York, NY: McGraw-Hill

Swinton, P. A., Stewart, A. D., Keogh, J. W., Agouris, I., \& Lloyd, R. (2011). Kinematic and kinetic analysis of maximal velocity deadlifts performed with and without the inclusion of chain resistance. The Journal of Strength \& Conditioning Research, 25(11), 3163-3174.

Tillin, N. A., Pain, M. T., \& Folland, J. P. (2012). Short-term training for explosive strength causes neural and mechanical adaptations. Experimental physiology, 97(5), 630-641.

Wilson, G. J., Newton, R. U., Murphy, A. J., \& Humphries, B. J. (1993). The optimal training load for the development of dynamic athletic performance. Medicine and science in sports and exercise, 25(11), 1279-1286. 